\title{
"Going into the unknown": Experiences of male patients in secure settings during environmental transition
}

Running title: Forensic patients in transition

Jacqueline H. Parkes MMedSci BA(Hons) PG Cert PG Dip RMN PhD ${ }^{1}$

Michelle Pyer BA (Hons) $\mathrm{PhD}^{1}$

Alison Ward BA(Hons) $\mathrm{MSc}^{1}$

Colin Doyle MBA MSc BSc RNMH Cert MHS ${ }^{2}$

Geoffrey L. Dickens RMN BSc(Hons) PGDipN MA PhD *1,3

*Corresponding author

Prof G.L. Dickens

St Andrew's Academic Centre

King's College London Institute of Psychiatry

Northampton

NN1 5DG

Telephone: +44 1604616362

Facsimile: +44 1604616158

${ }^{1}$ Centre for Health and Wellbeing Research, University of Northampton, Boughton Green Road, Northampton. NN2 7AL. UK.

${ }^{2}$ Cygnet Healthcare, Graveley Road, Stevenage, Hertfordshire. SG1 4YS. UK.

${ }^{3}$ St Andrew's Academic Centre, King's College London Institute of Psychiatry, Northampton. NN1 5DG. UK.

JHP: made a substantial contribution to conception and design, analysis of data and revised the article for important intellectual content

MP: made a substantial contribution to acquisition of data and analysis of data

AW: made a substantial contribution to analysis of data and drafting of the article

CD: made a substantial contribution to acquisition of data and analysis of data

GLD: contributed to conception and design, made a substantial contribution to acquisition of data, analysis of data, drafting of the article, and revised the article for important intellectual content

All authors had final approval of the version to be published. 


\begin{abstract}
Little is known about the experiences of male patients in secure mental health and intellectual disability units during environmental transition. We interviewed patients before $(\mathrm{N}=9)$ and after $(\mathrm{N}=8)$ a side-to-side security transition from medium-secure wards in an older building to new wards in a purpose-built building. We inquired about transitional experiences in general and about this transition specifically. We examined interview transcripts and field-notes using thematic analysis and collated routine outcomes data to gauge whether transition had obvious positive or negative effects. Qualitative analysis indicated three major themes (information, transition, and behaviour) and five overlapping sub-themes (positive information sharing \& consultation, concerns \& anxieties about lack of information, life change \& opportunity, home \& sense of belonging and potential conflict. Outcomes data indicated little obvious change between first and second interviews. Expressed concerns of patients around transition were largely about tangible and practical issues including changes to rules including smoking and prohibited items. Results highlight the need for the development of supportive patient inclusion strategies, consistent and transparent communication processes, and a published timeframe during the planning and implementation phases of all types of transitional moves, including the side-to-side transfer of residents between accommodation buildings.
\end{abstract}

Keywords: Forensic psychiatry, inpatients, qualitative research, life change events, men 


\section{INTRODUCTION}

Various forms of transition are inevitable across the life span that require personal adjustment, and may significantly alter an individual's roles, relationships, routines and assumptions (Schlossberg, 1984; Schlossberg et al, 1989; Chickering et al, 2002). Some transitions involve predictable or inevitable life events; others may be unexpected or arise in situations of extreme hardship and stress. For some individuals, and at some points in time, change presents new and exciting opportunities to be positively embraced, while for others, and at other times, it may lead to feelings of uncertainty, insecurity, increased anxiety, and a sense of loss (Schlossberg, 1981). The potentially negative effects of anticipated transition can often be ameliorated by adequate preparation, while unanticipated transition can involve sudden onset or crisis. Transition requires the individual to draw on their personal resources in order to adjust to the perceived opportunity or threat that is being presented. Every person responds differently to change and, within individuals, the consequences of transition will differ depending both on its nature and the developmental point at which it occurs. The human ability to adapt to change has been wellresearched and has given rise to two major theoretical traditions. The differential vulnerability argument suggests that either the individual's disposition or their social location largely determines the impact of the transition event (Cohen \& Wills,1985); while the trait approach advocates that events vary in their degree of stressfulness depending on their unpredictability, undesirability, uncontrollability and magnitude (Thoits, 1995). The role, history and presence of pre-existing levels of stress can also significantly determine the individual's ability to adapt to transition (Wheaton, 1990).

\section{Transition in Forensic Mental Health Services}


People with mental disorder are affected by the same transitional experiences which affect the wider population; however, they may find that their ability to cope with change has been compromised (World Health Organisation, 2010; World Health Organisation, 2011). Some other transitional experiences may be unique to individuals with mental disorder and arise from their mental illness or intellectual disability or as a consequence of their use of mental health services. People who are resident in inpatient Forensic Mental Health Services may find that they periodically experience planned or unplanned transition due to changing individual security needs, service reconfiguration, or funding changes. However, while research has identified how security and risk-related factors change for patients ${ }^{1}$ during transition (Collins \& Davies, 2005; Department of Health, 2010; Kennedy, 2002; Tighe \& Gudjonnsson, 2012) only a very limited number of studies have examined transition within or between forensic services from the perspective of patients.

The representation of forensic mental health patient's views during transition is important because they may hold life experiences which can reflect living in a constant atmosphere of uncertainty and unpredictability. Such experiences, when compounded by limited social and communications skills, active psychiatric symptomatology, and environmental stressors including the institutional regime and ward atmosphere, may have a disproportionate destabilising effect. Frequent lifelong exposure to stigmatising and devaluing conditions may lead individuals in this group to view themselves negatively and to have low future expectations (Dagnan, 2008). Consequently, patients in secure care can demonstrate an increased

\footnotetext{
${ }^{1}$ The term 'patient' is used in this paper rather than 'service user' because the corresponding author was the author of a recent study (Dickens et al, 2011) of terminological preference in the current study setting where this term 'patient' was preferred over all others including 'service user'.
} 
vulnerability to low mood and are less able to cope effectively with stressful situations. Requiring care that is relatively routine and consistent, the prospect of environmental upheaval in the form of an accommodation move could prove to be emotionally demanding on those who are unable to cope or adapt. Therefore, the management of change in secure mental health services should be considered carefully in order to promote successful adaptation to ensure effective transition for socially vulnerable patient groups. In the current study we were presented with a rare opportunity to explore the views and perceptions of patients resident in one of two medium secure wards (one for patients with mental disorder and one for those with an intellectual disability), and clinical staff during a period of environmental transition, specifically two 'whole ward' side-to-side security transfers. Interviews also offered an opportunity to discuss patient participant's forensic mental health service-related transitional experiences more widely.

\section{Aims and objectives}

In developing this study our aim was to develop new insights into the experience and effects of a change of space and environment for people who have a mental disorder and/or an intellectual disability, particularly when that change is in the context of a side-to-side security transfer from one medium secure setting to another. We sought to gather the views of patients about their past transitional experiences and of their expectations and experiences of change prior to, and following, the planned transfer of services into a newly built medium secure building. We aimed to ascertain whether the change described by patients was reflected in 'objective' routine outcomes data.

\section{MATERIALS AND METHODS}




\section{Setting and participants}

The study was conducted at St Andrew's Hospital, Northampton, UK, a charitable-sector provider of secure mental healthcare. In November 2010, patients and staff from two medium secure wards for adult men, one (Ward A) for those with mental disorder and one (Ward B) for those with an intellectual disability in the mild to moderate range, were relocated from two wards in the main hospital building (built 1838) into two wards of a newly built medium secure service comprising 128 beds in eight wards. Of note, a high-level managerial decision had been taken that the new building would be a non-smoking environment in all areas within the secure perimeter. As all outdoor areas such as courtyards and garden areas are within the secure perimeter then effectively the service became a non-smoking service. Patient participants were drawn from among the patient groups on Ward A (four) and Ward B (five). Mean age of patients was 31 years (range 24 to 51 years, S.D. $=9$ years) and had primary diagnoses of schizophrenia $(n=3)$, personality disorder $(n=1)$ and intellectual disability in the mild to moderate range $(n=5)$. Six patients described their ethnicity as 'White British', two as 'Black British' and one as 'Asian British'). Staff from Ward B (Ward A was not able to release staff) including members of psychiatry, psychology, nursing and occupational therapy professions participated.

\section{Procedure}

Ethical approval to conduct the study was sought and received from Northampton NHS Research Ethics Committee (10H046/69). Six weeks prior to the planned move two researchers (GD/MP) attended community meetings on both ward A and B to listen to patients views about the planned transition, to inform patients about the study and to ask them to consider participating in two interviews, one prior to the move and one six months later. Staff were also offered the 
opportunity to participate in a focus group about the planned move. Patients who expressed an interest in participating in the study were provided with a full written and verbal explanation before informed consent was taken. Responsible clinicians and clinical teams were consulted about each individual's capacity to consent and no one who was judged to lack capacity was recruited. Patient participants were offered a small payment ( $£ 10$ per interview) in respect of their time and input into the study.

\section{Materials}

Interviews were conducted by two researchers (Ward A: GD/MP; Ward B CD/MP) using a semistructured schedule. In the first round of interviews this comprised questions, items, prompts and cues designed to elicit accounts of prior experience of transition to, and within, forensic mental health services, and of current experience of the planned transition including any preparation or activities that they had received to help them adjust. The interview schedule used in the second round of interviews utilised a similar format to that of the first, but incorporated modified items to draw out the experiences of the recent transition. Field notes were also taken during community meetings on each of the two wards. Interviews with patients were audio-recorded where consent was given to do so and, in other cases, extensive and detailed notes were taken. Audio-recordings were transcribed and preliminary analysis of first round interviews was conducted to inform the precise content of the semi-structured questionnaire used in the second round of interviews. Participating patient's medical records were also accessed, with their consent, to extract routinely collected data to triangulate accounts of patient experience with 'objective' clinical data. In particular, we accessed routinely recorded outcomes data from the HoNOS-secure (Dickens et al, 2007) in order to identify any major changes in levels of 
aggression and self harm. A focus group with staff was conducted, audio-recorded and transcribed. Staff participants were formally consented to participate, ground rules were set for the group and a semi-structured schedule was used as the basis of discussion.

\section{Data Analysis}

A process of thematic analysis was employed to develop a comprehensive representation of the experience, meaning and reality of a side-to-side transition within a medium secure mental health service from participants' narrative accounts. We gathered routinely collected outcomes data and staff accounts to provide an 'objective' counterpoint view; change on ratings before and after the transfer were analysed using the non-parametric Wilcoxon signed ranks test. For qualitative data, analytical processes described by Braun and Clark (2006) were followed. We adopted an essentialist/realist perspective towards data analysis indicating our underlying assumption that the language used by participants would articulate the meaning, and experience, of transition with little need for deeper interpretation. Transcripts and field notes were read recursively throughout the study and during subsequent analysis. Initial open coding of narrative data was undertaken manually by two members of the research team and involved identification of basic semantic segments of the interview data, each transcript being retained as an individual data source at this point. Subsequently, segments from multiple data sources were combined into themes, with further re-coding occurring following review and discussion by the team.

\section{RESULTS}

In total nine patient participants were interviewed six weeks prior to the transition event (round one interviews). Six months later all but one participant was still resident in the service (one had been transferred out of area) thus eight were re-interviewed (round two interviews). The thematic 
analysis of 17 interviews suggested that patient interviewee experiences fell broadly under three major themes comprising five overlapping sub-themes (see Fig 1). Theme one related to information and comprised sub-themes relating to positive information sharing \& consultation and concerns \& anxieties specifically about lack of information. Theme two related to transition and comprised sub-themes of life change \& opportunity, home \& sense of belonging and also encompassed the concerns \& anxieties sub-theme; however, transitional concerns tended to be largely about environmental issues rather than information ones. Theme three related to behaviour and this was also related to the sub-theme of life change $\&$ opportunity and a final sub-theme potential conflict. This last sub-theme also overlapped with both the information and environmental elements of the concerns \& anxieties sub-theme. We agreed on these themes based on the whole narrative data set and did not find that different themes substantially reflected differential accounts based on a mental health or learning disability diagnosis.

$<<$ Insert Fig 1 about here $>>$

\section{Theme one: Information}

Information was a key theme of importance for participants. They discussed their informational needs both in relation to the current move (anticipated and realised issues) and also drew from their previous experiences of transitioning to their current unit. Many participants had experienced that the amount and detail of information provided prior to their current placement had been sparse and somewhat variable highlighting that information sharing and consultation were important to them (sub-theme 1). Two participants reported that they had not been informed about their transfer into the unit from their previous residence until the day that it occurred. All patient participants in round one interviews were aware of the planned side-to-side 
transitional transfer to new accommodation, and of the precise date on which it would occur. All participants also informed the project team spontaneously during the first round interviews that the new building would have a no smoking policy, and bedrooms would have en suite bathrooms as opposed to the communal facilities in situ. Participants also reported that some information at been shared about the planned move during ward community meetings; however, individual ad hoc reports between participants and clinical, managerial or advocacy staff appeared to differ in the consistency of information provided. Some participants appeared to have gleaned extensive information about their new ward and facilities from nursing staff; while others appeared to be less clear on what to expect. It was apparent that, while senior managers had visited ward community meetings prior to the side-to-side transition to share information about the planned move, some participants felt that their questions or concerns had remained unanswered. In addition, some ward staff echoed that they themselves had been provided with insufficient information to satisfactorily answer patient's information requests.

'There was a woman came down to answer questions at the last community meeting but she didn't know the answers.' (P2, Ward B)

'... the patients have been asking for some time whether they can go and visit the new build and I wasn't able to give them a clear response for that. And now it seems like there isn't going to be enough time for that.' (Staff member 3, Ward B)

There appeared to be a small, but appreciable, gap between ward staff accounts of their attempts to give patients information about the transfer and patient's reported satisfaction with the fullness of information received. For example, staff were able to spontaneously detail a number of interventions they had delivered to prepare patients for the move, including talking, showing photographs of the new building and its facilities, use of a 'countdown calendar' to the move, provision of information about rule changes relating to electrical equipment and storage of 
excess belongings, and provision of a plan of bedroom allocation. While some of this was verified by patient's own accounts, a number of patient participants insisted they had received insufficient information.

'I remember a month or two months ago they came with a board with the layout on [to allow patients to choose colours of furnishings]... most of us chose the same colours. [One patient asked if there was going to be carpet in the rooms and was told that some would have carpet and some would have wood]. I chose wood' (P2, Ward A).

The second information sub-theme was concerns \& anxieties and this largely comprised participant's statements outlining uncertainty, or indeed unwelcome certainty, around a small number of delimitated, practical issues. For example, a number of the participants in round one interviews felt that information about issues including the size of television sets and the types of electrical equipment allowed in bedroom areas had been lacking in detail. Other concerns related to planned changes to smoking policy and bedroom allocation and access.

'Manager picked bedroom. We're not happy with it none of us.' (P5, Ward B)

[in relation to smoking policy change] 'We don't have a choice about being here. How would anybody feel about someone coming into your home and telling you what to do?' (P3, Ward A)

\section{Theme two: Transition}

Many of the participants discussed their experiences of first arriving at the current secure unit during round one interviews. For most, this change had been experienced as stressful, with commonly occurring reports of not knowing what to expect, worrying about fitting in and meeting new people.

\footnotetext{
'...going into the unknown isn't it?' (P2, Ward B)
} 
Sometimes change was associated with, or was followed by, an emerging sense of opportunity; which could relate to the chance to use better facilities or improved access to treatment; but occasionally, this is associated a longer-term vision for life beyond secure care.

'Yeah I knew I was going to get better treatment than what I was getting in that place.' (P?, Ward A)

'... going for a walk round the grounds and that, and going to different sessions off the ward and stuff like that and I quite enjoy it here' $(P 1$, Ward $A)$

'... hopefully by the time I leave here I will be able to go back into the community and get myself a job and get my life back together. I am just focused now on the future and not dwelling on the past...' (P1, Ward A)

Patient experiences of adapting to change often appeared to relate to feeling accepted and settled within the new placement, which in turn, appeared to be associated with the inter-linked notions of home and sense of belonging.

'The first few days was very strange for me, it was trying to fit in, getting to know the staff, getting to know the other patients and for them getting to know me as well. It certainly was all quite strange for me.' (P4, Ward A)

'It was just so very difficult for me to communicate just to know what to say or anything... I didn't want to come out with the wrong thing and upsetting them.' (P1, Ward A)

Finally, and similarly to the information theme, the transition theme encompassed a number of concerns \& anxieties; which appeared to be more related to the environment both in a narrow local sense and from a wider perspective than within the theme of information. For example, some participants' initial transition to the site had been related to a reduced proximity to family and friends, and concerns about the potential impact of this on family contact. In terms of the current transition, several participants reported feeling initially dissatisfied following the move, 
as the full range of facilities had not been available in the first few weeks, leaving them with feelings of unmet need.

'I said I don't wanna go there because it's far from home.' (P5, Ward B)

'They moved us down when the building wasn't ready.' (P3, Ward A)

In addition, environmental issues relating to access to communal living areas and private bedrooms left some participants with a reduced sense of belonging and increased feelings of tension.

'... the lounge is a bit small, if you have an argument with another patient you have nowhere to go... nowhere to escape to.' (P2, Ward B)

'... if you ain't got no sessions everyone's got to stay in the lounge... They always have to like put telly on or find something else for us to do. We have to use initiative or staff... in the cupboard they've got games and that. I find it quite frustrating sometimes cos I like it when it's quite peaceful and when everyone's kicking off or arguing or stuff like that it pisses me off and I find it frustrating. When it's quiet and everything else than I find it not too bad.' (P2, Ward B)

\section{Theme 3: Behaviour}

Many of the participants reported that their behaviour, or other functioning, had not appreciably altered post side-to-side transition. The majority reported feeling generally positive about the move. For some, there had been a brief period of adjustment, but they had subsequently settled well into their new surroundings and routines; however, staff had reported prior to the move that individuals in the wider patient group appeared to be coping less well with the move, with some requiring more regular reassurance.

'I think there are some patients who have found change very difficult ... for them it's always something that's going to cause great anxiety isn't it? Also I think it's the changing of the environments is stressful.' (Staff member 1, Ward B) 
'... they are losing ... their safety net' (Staff member 6, Ward B).

For some, the transition had facilitated change \& opportunity which the interviewee believed had manifested in pro-social behaviour change, such as being able to access more sessions and activities. A minority of interviewees also felt that the introduction of the 'no smoking' policy had had a positive effect on the behaviour of some residents, encouraging them to reduce or even quit their habit. For others, it had improved some patients more problematic behaviours associated with their tobacco use.

'Oh it's made quite a lot of difference to me... I'm able to do more things... I can do more lessons...' (P2 Ward A)

'No trouble in here since I've been here. Nobody scrounges off you for tobacco...' (P4 Ward A)

'... to be fair I know I shouldn't talk about other patients but really they have done fantastic. Not moaning "I need a fag" "I want to go out". Really been good.' (P1 Ward B)

For the majority of residents who smoked prior to the move, they continued to do so post move, generally outside of the secure perimeter while taking ground leave.

'... if you've got leave to go out in the grounds then you can do that. When we're in the grounds we're allowed to have a smoke.' (P2, Ward A)

For one patient, being caught smoking in a public area [bathroom] in contravention of unit policy, and faced with the possibility of losing his privileges and level status, provided the motivational impetus to stop smoking altogether.

'I decided after I got caught once that it wasn't worth losing my level 5 [risk rating level] over.'

Reference to the routinely collected outcomes data immediately prior to and post side-to-side transition indicated no significant improvement or deterioration in behaviours linked to 
aggression $(Z=0.137, p>0.05)$, self injury $(Z=-1.342, P>0.05)$ or to living conditions $(Z=-1.414$, $\mathrm{p}>0.05$ ) in the behaviour of the residents.

\section{DISCUSSION}

In the current study we described the transition-related experiences of male medium secure patients. The study focused on experiences related to the transition to their current placement and also on experiences prior to and following the transfer of patients to new, purpose-built accommodation. We were therefore able to gather rich and detailed data about the phenomenon at a time pertinent to a group of patients experiencing that phenomenon. Thematic analysis suggested that this group's experiences related to information, transition and behaviour. Within these broad themes a number of related sub-themes suggested that participants experience was shaped by information sharing and consultation, that they had concerns and anxieties related to informational and environmental aspects of transition; that there was a sense of need for home and belonging within the medium secure environment; and that there was the potential both for opportunity and change, and conflict during the transitional period. We do not propose to hypothesise about causal directionality within these related themes; however, we do suggest that many of these themes are inter-related and indeed overlapping. There was a tangible recognition among staff of the transitional experience and its potential effects, and an equal desire to plan to ameliorate negative effects and magnify positive experiences. 'Objective' outcomes data revealed little apparent change in status over the transitional period, but this appeared to mask a depth of rich lived experience. The aim of our study was to provide a description of this experience to inform future transitional planning; it was not our intention to test theoretical hypotheses although a number of observations can be made within the context of that theory. 
The current study explored both forensic mental health service-related transitional experiences in general and a specific side-to-side security transition in particular. Accounts of general transitional experiences indicated a greater potential for distress during, for instance, admission to services or transfer to a new service a distance from the existing placement without sufficient preparation. In contrast, discussions focussing on the side-to-side security transition rarely elicited emotional responses; rather it was seen as an inevitable change. This resulted in a focus on changes to day-to-day routine. This evidence could be used to support a trait approach (Thoits, 1995) to transition-related vulnerability in that the side-to-side transition was evidently not unpredictable (patients were made aware of the forthcoming change and the timeframe for this), was not perceived as especially undesirable per se (the environmental advantages resulting from the transition were clearly articulated), nor as of great magnitude or consequence (long term outcomes were not a focus for discussion, rather their daily living experiences).

Elements of uncontrollability, for example related to concurrent introduction of a no smoking policy or lack of certainty around rules, did appear to have the potential to lead to conflict. However, this does not rule out a place for differential vulnerability theory in this arena (Cohen \& Wills, 1985, Wheaton, 1990). Quite clearly some individuals reported adverse experience of prior transition-related experience, and there is a need to make a careful individual assessment of vulnerability prior to any planned transition.

We found that some men in our study described positive experiences related to the current transition, and a sense of potential for positive growth and change and this is congruent with theoretical standpoints on transition (Schlossberg, 1981). Similarly, participants valued the sense of home and belonging offered by the secure therapeutic environment, a finding also supportive 
of previous work (Blunt \& Dowling, 2006). However, there was also a sense of uncertainty, particularly around changes to rules and privileges. Other studies have noted a tendency among people in secure care to concentrate on 'toeing the line' or focussing on earning privileges rather than truly addressing the reasons for their admission into secure care in the first place (ParryCrooke et al, 2000, Walker et al, 2005). This focus on the day-to-day, rather than the long term was certainly evident in some of our participants' focus on concrete issues around rules and privileges, for example where they discussed the size of television that they were allowed. Some participants also focussed on the potential for them to increase their access to the grounds of the new building by earning this privilege through the levels system that was put into place.

Potential changes to rules were seen as threatening within the context of the transition in terms of impacting on daily routine and activity. This was also true of a move towards a non smoking policy. We know that psychiatric inpatients are likely to smoke (Smith, 2008) and that they are likely to be supportive of policies which facilitate their continued use of tobacco (ibid). We also know that no smoking policies can be introduced in secure environments with little apparent adverse effect on objective measurements of conflict including seclusion or use of psychotropic medication (Cormac et al, 2010). Such initiatives, however, tend not to attempt any objective measurement of satisfaction with a ban, and there is little evidence that patients view such prohibitions as positive, rather they are seen as an intrusion to be tolerated. Participants in our study rarely described themselves as non-smokers following transfer to the new non-smoking unit and most continued to smoke when outside of the secure perimeter. Clearly, some will take this as evidence that greater efforts are required to control access to tobacco, including off-ward access. Some staff saw the clear benefits of a no smoking policy in that the daily routine no longer revolved around hourly access to the smoking shelter, resulting in less disruption to 
therapeutic activity. We suggest that medium secure patients facing transition appear to appreciate certainty around any change in particular in relation to how this may affect, adversely or otherwise, the activities and resources that they view as privileges. This suggests that, at least, equal attention should be paid to planned change to these items within the course of transition as it is to physical and environmental aspects. This will require considerable forward planning on behalf of managerial and clinical staff, consultation with patients, and a strategy to disseminate plans. It may benefit patients undergoing transition to have some certainty about changes to rules, although this of course must be balanced with security-related issues. It is essential that this is communicated to patients clearly and openly. The study found that staff were able to share a number of activities which they had undertaken to prepare patients for the transition, but these rarely focused on rule change.

\section{Conclusion}

As human individuals journey through life, it is inevitable that they will experience a number of transitional experiences that require some degree of personal adjustment or adaptation in order to cope with the change. For the most part, it appears that the effects of anticipated transitions can often be mediated against through the implementation of effective communication strategies prior to the expected event; however, where the transition is less predictable or unanticipated, the response is more likely to generate feelings of uncertainty and anxiety and lead to possible crisis. For individuals who reside in secure mental health services, their ability to successfully cope with change has already been compromised by their psychological vulnerabilities, and yet, their transitional histories often record extensive movement between services. For this group of patients, the effects of unanticipated general transitional experiences, such as transfer between services, has the potential for greater distress and behavioural response, than a planned change. For the longer-residents of 
secure institutions, the effects of an environmental change, in the form of a side-to-side accommodation move, appears to have elicited limited emotional response. This can clearly be attributed to the information that individuals received prior, during, and post move; and their involvement in some planning activities in preparation to the move. However, where there was the 'sudden' unexpected introduction of policy changes to daily routines, the patients did experience both anxiety and behavioural changes, which had the potential to result in conflict. Overall, the findings of this study demonstrates that the residents of secure institutions with their familiar 'strict routines, regimes, and regulations' (Cashin et al, 2010), which they have grown to regard as 'home', still require individual assessments of psychological vulnerability, adequate preparation and ongoing social support before, during, and after any transitional experience. This in turn highlights the need for the development of supportive patient inclusion strategies, consistent and transparent communication processes, and a published timeframe during the planning and implementation phases of all types of transitional moves, including the side-to-side transfer of residents between accommodation buildings.

\section{ACKNOWLEDGMENTS}

The study was funded by St Andrew's Healthcare. 


\section{REFERENCES}

Blunt, A. \& Dowling, R. (2006) Home. London: Routledge.

Braun, V., \& Clarke, V. (2006) Using thematic analysis in psychology. Qualitative Research in Psychology, 3, 77-101.

Cashin, A., Newman, C., Eason, M., Thorpe, A. \& O’Discoll, C. (2010) An ethnographic study of forensic nursing culture in an Australian prison hospital. Journal of Psychiatric and Mental Health Nursing, 17, 39-45.

Chickering, A.W.S., \& Schlossberg, N.K. (2002) Getting the most out of college. Upper Saddle River, NJ: Prentice-Hall.

Cohen, S.W. \& Wills, T.A. (1985) Stress, social support, and the buffering hypothesis.

Psychological Bulletin, 98, 310-357.

Collins, M.D. \& Davies, S. (2005) The Security Needs Assessment Profile: a multidimensional approach to measuring security needs. International Journal of Forensic Mental Health, 4, 3952.

Cormac, I., Creasey, S., McNeil, A., Ferriter, M., Huckstep, B. \& D’Silva, K. (2010) Impact of a total smoking ban in a high secure hospital. The Psychiatrist, 34, 413-417.

Dagnan, D (2008) Psychological and Emotional Health and Well-Being of People with Intellectual Disabilities. Tizard Learning Disability Review, 13, 3-9.

Department of Health (2010) Relational security. London: Department of Health.

Dickens, G., Langé, A., \& Picchioni, M. (2011) Labelling people who are resident in a secure forensic mental health service: user views. Journal of Forensic Psychiatry and Psychology, 22, 885-894.

Dickens, G., Sugarman, P., \& Walker, L. (2007) HoNOS-secure : A reliable outcome measure for users of secure and forensic mental health services. Journal of Forensic Psychiatry and Psychology, 18, 507-514.

Kennedy, H.G. (2002) Therapeutic uses of security: mapping forensic mental health services by stratifying risk. Advances in Psychiatric Treatment, 8, 433-443.

Parry-Crooke, G., Oliver, C., \& Newton, J. (2000) Good girls: surviving the secure system. London: University of North London.

Schlossberg, N.K. (1981) A model for analyzing human adaptation to transition. Counseling Psychologist, 9, 2-18. 
Schlossberg, N.K. (1984) Counseling adults in transitions. New York: Springer.

Schlossberg, N.K., Lynch, A.Q., \& Chickering, A.W. (1989) Improving higher education environments for adults. San Francisco: Jossey-Bass.

Smith, J. (2008) Exploration of in-patient attitudes towards smoking within a large mental health trust. The Psychiatrist, 32, 166-169.

Thoits, P.A. (1995) Stress, coping and social support processes: Where are we? What next? Journal of Health and Social Behavior, Extra Issue, 53-79.

Tighe, J. \& Gudjonsson, G. H. (2012) See, Think, Act Scale: preliminary development and validation of a measure of relational security in medium- and low-secure units Journal of Forensic Psychiatry and Psychology, 23, 184-199.

Walker, M., Barclay, A., Malloch, A., Kendrick, A., Hunter, L. \& Hill, M. (2005) Secure accommodation in Scotland: its role and relationship with 'alternative' services. Edinburgh: Scottish Executive Education Department.

Wheaton, B. (1990) Life transitions, role histories, and mental health. American Sociology Review, 55, 209-223.

World Health Organisation (2010) Fact sheet No 220 - Mental health: Strengthening our response. http://www.who.int/mediacentre/facts (accessed 11/01/2012)

World Health Organisation (2011) Fact sheet No 352 - Disability and health. http://www.who.int/mediacentre/facts (accessed 11/01/2012) 


\section{Figure Legends}

Figure 1: Forensic patients in transition: thematic analysis 


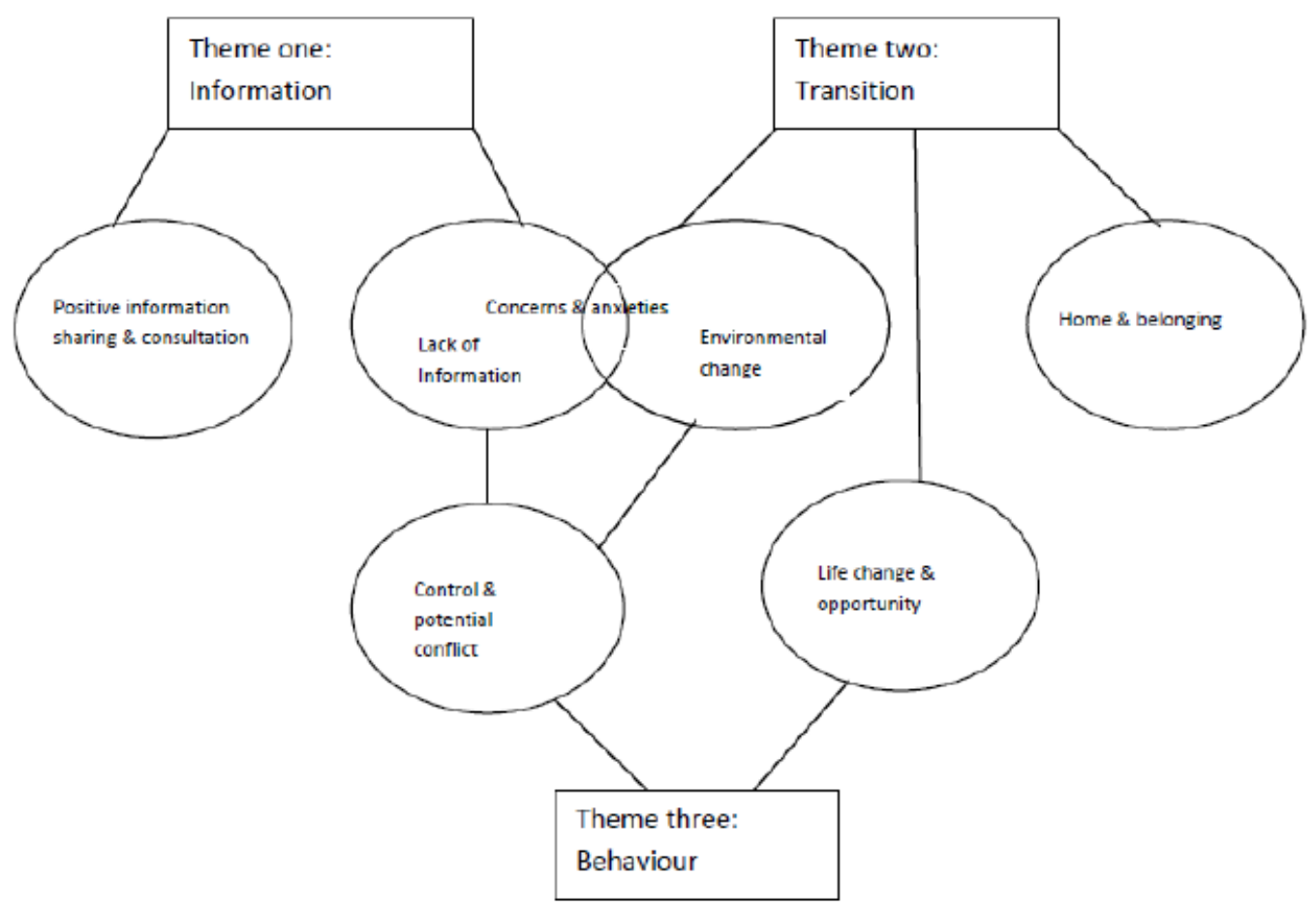

